Revista Brasileira de Agricultura Irrigada v.7, no . 2, p. 146 - 156, 2013

ISSN 1982-7679 (On-line)

Fortaleza, CE, INOVAGRI - http://www.inovagri.org.br

DOI: $10.7127 /$ rbai.v7n200002

Protocolo 002.13 - 23/11/2012 Aprovado em 30/04/2013

\title{
DESENVOLVIMENTO RADICULAR DA BERINJELA IRRIGADO E DE SEQUEIRO EM DIFERENTES FORMAS DE CULTIVO
}

\author{
Gabriel Queiroz de Oliveira ${ }^{1}$, Pedro Luiz Nagel ${ }^{2}$, Adriano da Silva Lopes ${ }^{3}$, Francielli \\ Schwerz ${ }^{4}$, Paula Ariana da Silva ${ }^{4}$, Raimundo Rodrigues Gomes Filho ${ }^{5}$
}

\begin{abstract}
RESUMO
A berinjela é uma cultura bastante apreciada e cultivada, no entanto, existem poucas informações sobre seu manejo irrigado, principalmente quando se diz respeito a profundidade efetiva do sistema radicular para correta irrigação. Com o objetivo de avaliar a densidade e a distribuição de raízes de berinjela submetidos á aplicação e ausência de irrigação por método gotejamento fornecendo adubação química e orgânica, realizou-se um experimento em Aquidauana-MS. O delineamento utilizado foi em blocos casualizados, com parcelas subdivididas, utilizando quatro blocos. Os tratamentos empregados nas parcelas corresponderam a berinjela irrigada e não irrigada e as subparcelas foram compostas de adubação orgânica e química. As irrigações foram realizadas com base na evaporação do tanque Classe A e por meio de tensiômetros. As adubações química e orgânica, utilizando ureia e organosuper $^{\circledR}$ respectivamente, foram realizadas aos 45, 60 e 75 dias após o transplante (DAT). As coletas das raízes foram realizadas aos 113 DAT, para isso utilizando um trado tipo caneco, e retirando amostras em três distâncias do caule da planta $(0,0,0,2$ e 0,4 $\mathrm{m})$ e em quatro profundidades do solo $(0,00-0,10 ; 0,10-0,20 ; 0,20-0,30$ e $0,30-0,40 \mathrm{~m})$ sendo em seguida, lavadas, levadas a estufa e pesadas em balança analítica. Conclui-se que a profundidade efetiva do sistema radicular da berinjela irrigada por gotejamento por ambas as adubações é no máximo de $0,20 \mathrm{~m}$.
\end{abstract}

Palavras chave: Solanum melongena L., profundidade efetiva, gotejador, densidade de raiz.

\section{ROOT SYSTEM OF EGGPLANT IRRIGATED AND DRYLAND CROPPING IN DIFFERENT SYSTEMS}

\begin{abstract}
\footnotetext{
${ }^{1}$ Doutorando em Agronomia, FCA/UFGD, CEP: 79804-970, Dourados, MS, Brasil. gabrielqo@ hotmail.com. ${ }^{2}$ Mestrando em Agronomia, UUA/UEMS, CEP: 79200-000, Aquidauana, MS, Brasil. nagelpedro@yahoo.com.br.

${ }^{3}$ Professor Adjunto, UUA/UEMS, CEP: 79200-000, Aquidauana, MS, Brasil. lopes@uems.br

${ }^{4}$ Mestranda em Engenharia Agrícola, FCA/UFGD, CEP: 79804-970 Dourados, MS, Brasil. fran_schwerz_88@hotmail.com; paulariana@ @otmail.com

${ }^{5}$ Professor Adjunto, UFG, Campus Jataí, CEP: 75800-000, Jataí, GO, Brasil. rrgomesfilho@ hotmail.com
} 


\section{DESENVOLVIMENTO RADICULAR DA BERINJELA IRRIGADO E DE SEQUEIRO EM DIFERENTES FORMAS DE CULTIVO}

Aiming to evaluate the density and distribution of roots of eggplant with supply of organic fertilizer and chemical application will be submitted and no drip irrigation method in ultisol, there was an experiment in Aquidauana-MS. The design was randomized blocks with split plots, using four blocks. Treatments used in the plots corresponded to eggplant and without irrigation and the subplots consisted of organic fertilizer and chemical industries. Irrigation was performed based on the evaporation of the Class A pan and through tensiometer. The organic and chemical fertilization using urea and organoeste respectively, were performed at 45, 60 and 75 days after transplanting (DAT). The gathering of roots were done at 113 DAT for this using an auger hole, and taking samples at three distances from the plant stem (zero, 0.2 and $0.4 \mathrm{~m})$ and four soil depths $(0.00-0.10,0.10-0.200 .20-0.30$ and $0.30-0.40 \mathrm{~m}$ ) were then washed, taken to oven and weighed on an analytical balance. It follows that the effective depth of the root system of drip irrigated eggplant by both fertilization is a maximum of 0.20 m.

Keywords: Solanum melongena L., root depth, dripper, root density.

\section{INTRODUÇÃO}

O sistema radicular da berinjela (Solanum melongena L.) é vigoroso e profundo, atingindo profundidades superiores a $100 \mathrm{~cm}$, embora a maioria das raízes se concentre mais superficialmente. Esta é uma planta originária de regiões de clima tropical e subtropical, sendo uma das culturas oleráceas mais exigentes em calor, como também em luminosidade (FILGUEIRA, 2003).

A literatura refere-se à berinjela como planta altamente produtiva, que requer elevadas adubações, porém equilibradas, para assegurar as quantidades necessárias de nutrientes para o crescimento das plantas e elevadas produções, confirmando a escassez de estudos sobre os níveis de fertilizantes e adubos orgânicos (FILGUEIRA, 2000; FILGUEIRA, 2003; RIBEIRO et al., 1998). Nesse sentido, estudos sobre aspectos morfológicos da planta, como a distribuição do sistema radicular sob diferentes condições de manejo, são interessantes, na medida em que possam auxiliar e fornecer informações para a realização dos principais tratos culturais, como irrigação e adubação da cultura.

Os cálculos da lâmina líquida ou bruta de irrigação para qualquer cultura levam em consideração a profundidade efetiva do sistema radicular (BERNARDO, 2008). O uso equivocado da profundidade efetiva do sistema radicular pode trazer erro considerável a um projeto de irrigação, de maneira que o estudo do sistema radicular das espécies vegetais utilizadas na agricultura, sua distribuição, extensão e atividade, é incontestável e fundamental para o entendimento científico da produção agrícola (FRACARO; PEREIRA, 2004). No entanto, normalmente, os estudos do sistema radicular das culturas são voltados para as condições de sequeiro, quando não são direcionados para irrigação por gotejamento. Em se tratando de irrigação localizada, como gotejamento, apenas o conhecimento da profundidade efetiva do sistema radicular não é suficiente para inferir as zonas de absorção de água e nutrientes, uma vez que a geometria de distribuição de água no solo nesse tipo de irrigação é de caráter multidimensional (COELHO; SANTOS; COELHO FILHO, 2005). Os mesmos autores salientaram que para a irrigação localizada, é necessário conhecer tanto a profundidade efetiva como a distância horizontal efetiva das raízes em relação à planta.

A adubação orgânica permite melhora na fertilidade, além de ser 


\section{DESENVOLVIMENTO RADICULAR DA BERINJELA IRRIGADO E DE SEQUEIRO EM DIFERENTES FORMAS DE CULTIVO}

excelente condicionador de solo, melhorando suas características físicas, químicas e biológicas, como retenção de água, agregação, porosidade, aumento na capacidade de troca de cátions, aumento da fertilidade e aumento da vida microbiana do solo (MIYASAKA et al., 1997).

\section{MATERIAL E MÉTODOS}

O experimento foi desenvolvido em Aquidauana-MS, com coordenadas geográficas $20^{\circ} 20^{\prime} \mathrm{Sul}, 55^{\circ} 48^{\prime}$ Oeste. O clima da região, segundo a classificação de Köppen, é descrito como Aw, definido como clima tropical quente sub-úmido, com estação chuvosa no verão e seca no inverno e precipitação pluviométrica média anual de $1200 \mathrm{~mm}$, com chuvas concentradas nos meses de outubro a
O objetivo deste trabalho foi avaliar a distribuição do sistema radicular analisando a densidade de raiz da berinjela, irrigado por gotejamento e de sequeiro, com aplicação de adubação química e orgânica em cobertura.

fevereiro e, a altitude média de $174 \mathrm{~m}$ e o solo estudado foi classificado como Argissolo Vermelho-Amarelo (EMBRAPA, 2006).

As características químicas do solo foram determinadas na camada de $0,0-0,2$ $\mathrm{m}$ e 0,2-0,4 $\mathrm{m}$ e do adubo orgânico organosuper ${ }^{\circledR}$ estão apresentadas na Tabela 1. A adubação de plantio foi realizada diante da análise química do solo (Tabela 1) e de acordo com a recomendação de Ribeiro, Brune e Reifchneider (1998).

Tabela 1. Caracterização química de amostra do solo e do adubo orgânico organosuper ${ }^{\circledR}$.

\begin{tabular}{ccccccccccc}
\hline $\begin{array}{c}\text { Profundidade do } \\
\text { solo } \\
\mathrm{m}\end{array}$ & $\mathrm{pH}$ & $\mathrm{P}$ & $\mathrm{MO}$ & $\mathrm{K}$ & $\mathrm{Ca}$ & $\mathrm{Mg}$ & $\mathrm{Al}$ & $\mathrm{H}+\mathrm{Al}$ & $\mathrm{S}$ & $\mathrm{T}$ \\
\cline { 3 - 10 } $0,0-0,2$ & 6,8 & 92,5 & 2,5 & 0,4 & 4,5 & 1,0 & 0,1 & 1,6 & 5,9 & 7,5 \\
\hline $0,2-0,4$ & 6,7 & 486,0 & 3,0 & 0,6 & 5,2 & 1,2 & 0,1 & 1,8 & 6,9 & 8,8 \\
\hline Organosuper $^{\circledR}$ & 6,7 & 704,8 & 24,0 & 3,9 & 14,9 & 11,9 & - & - & - & - \\
\hline
\end{tabular}

A caracterização física do solo (Tabela 2), composta por densidade do solo, porosidade total, macro e microporosidade, foi realizada em camadas de 0,$1 ; 0,2 ; 0,3$ e $0,4 \mathrm{~m}$, utilizando-se metodologias descritas no manual de métodos da Embrapa (EMBRAPA, 1997).

Tabela 2. Caracterização física de amostra do solo.

\begin{tabular}{ccccc}
\hline \multirow{2}{*}{$\begin{array}{c}\text { Profundidade de solo } \\
\mathrm{m}\end{array}$} & $\begin{array}{c}\text { Densidade do solo } \\
\mathrm{g} \mathrm{cm}^{-3}\end{array}$ & \multicolumn{3}{c}{$\begin{array}{c}\text { Porosidade do solo } \\
\mathrm{cm}^{3} \mathrm{~cm}^{-3}\end{array}$} \\
\cline { 3 - 5 } & 1,48 & Macro & Micro & Total \\
\hline $0,00-0,10$ & 1,53 & 0,18 & 0,21 & 0,38 \\
$0,10-0,20$ & 1,53 & 0,15 & 0,2 & 0,35 \\
$0,20-0,30$ & 1,55 & 0,15 & 0,22 & 0,36 \\
$0,30-0,40$ & 0,13 & 0,24 & 0,37 \\
\hline
\end{tabular}




\section{DESENVOLVIMENTO RADICULAR DA BERINJELA IRRIGADO E DE SEQUEIRO EM DIFERENTES FORMAS DE CULTIVO}

A cultura utilizada foi à berinjela com espaçamento de $0,8 \times 1,2 \mathrm{~m}$, no qual cada bloco possuía $12 \mathrm{~m}$ de comprimento com oito linhas. A variedade de berinjela escolhida foi a "Ciça", sendo semeada em bandejas de poliestireno em estufa plástica e, no dia 27 de março de 2010, transplantada a campo.

O delineamento utilizado foi em blocos casualizado, em esquema de parcela subdividida, utilizando quatro blocos. As parcelas corresponderam à berinjela irrigada e não irrigada. As subparcelas foram compostas de adubação orgânica e química.

Adotou-se o sistema de irrigação por gotejamento sendo utilizados emissores com vazão de $4,5 \mathrm{~L} \mathrm{~h}^{-1}$ e pressão de serviço de $8 \mathrm{~m}$ c.a. instalados em cada linha, no qual cada gotejador continha um espaçamento de $0,40 \mathrm{~m}$. As irrigações foram realizadas, com base na evaporação do tanque Classe A e por meio de monitoramento da tensão de água do solo com o uso de tensiômetros, instalados a 0,15 e $0,30 \mathrm{~m}$ de profundidade. A irrigação foi sempre efetuada quando o potencial mátrico era igual ou inferior a $-30 \mathrm{kPa}$, com lâmina de irrigação para repor as perdas por evaporação estimada pelo tanque Classe A, sendo que o coeficiente do tanque (kp) foi de 0,7 e coeficiente de cultura (kc) foi conforme (ALLEN et al. (1998).

A adubação química de cobertura utilizou como fonte a uréia, fornecendo 15

\section{RESULTADOS E DISCUSSÃO}

A lâmina total aplicada (irrigação + precipitação) durante o experimento foi de $304,38 \mathrm{~mm}$, sendo que a precipitação correspondeu a $85,38 \mathrm{~mm}$. Estes foram controlados até 110 dias após transplante a campo.

Em relação à irrigação, observou-se diferença significativa para todas as distâncias do caule das plantas, nas duas primeiras profundidades. Para $\mathrm{o}$ fator gramas por planta, e a adubação orgânica de cobertura aplicou 45 gramas por planta do adubo orgânico organosuper ${ }^{\circledR}$, sendo realizada aos 45, 60 e 75 dias após o transplante.

A coleta do sistema radicular da berinjela foi realizada aos 113 dias após o transplante entre linhas de plantio, através de coletas a 0,$0 ; 0,2$ e $0,4 \mathrm{~m}$ do caule correspondendo a $\quad \mathrm{D}_{1} ; \quad \mathrm{D}_{2} \quad \mathrm{e} \quad \mathrm{D}_{3}$ respectivamente, e em quatro profundidades para cada distância: 0,000,$10 ; 0,10-0,20 ; 0,20-0,30$ e $0,30-0,40 \mathrm{~m}$, sendo descritos como $\mathrm{P}_{1} ; \mathrm{P}_{2} ; \mathrm{P}_{3}$ e $\mathrm{P}_{4}$. Para tal, foi utilizado um trado tipo caneco com $10 \mathrm{~cm}$ de altura e o volume coletado para cada amostra foi lavado em laboratório, com o auxílio de uma peneira com malha fina e colocado em saco de papel para secar em estufa a $65{ }^{\circ} \mathrm{C}$, durante 72 h. Em seguida pesados em balança analítica de precisão de 0,0001 g, sendo os dados expressos em gramas de massa seca de raiz por volume de solo $\left(\mathrm{g} \mathrm{dm}^{-3}\right)$. A profundidade efetiva do sistema radicular foi definida conforme a profundidade em que se concentravam $80 \%$ de peso das raízes.

De posse dos dados, foi realizada a análise de variância para a comparação das médias em relação a irrigação e adubação e, de superfície de resposta da densidade de raiz, em função da profundidade do solo e distância do caule, procedendo em esquema utilizando o software Statistical Analysis Systems (SAS, 1999).

adubação e a interação entre irrigação e adubação, somente houve diferença significativa na região mais superficial e próxima ao caule. Quanto à densidade de raiz submetida aos tratamentos irrigado e sequeiro, verificou-se que os valores foram muito superiores quando as plantas que receberam irrigação, porém somente a região mais superficial e próxima do caule teve diferença significativa, comprovando que a ação da lâmina de água influenciou em uma maior acúmulo de matéria seca de 


\section{DESENVOLVIMENTO RADICULAR DA BERINJELA IRRIGADO E DE SEQUEIRO EM DIFERENTES FORMAS DE CULTIVO}

raiz para a região citada (Tabela 3 ).

Para todas as distâncias do caule, as duas regiões mais profundas do solo $\left(\mathrm{P}_{3} \mathrm{e}\right.$ $\mathrm{P}_{4}$ ) não diferiram significativamente para os tratamentos irrigado e sequeiro (Tabela 3). Este resultado condiz com o que foram sugerido por Taiz e Zieger (2004) que, quando as camadas do solo estão pouco umedecidas, há redução no desenvolvimento de raízes superficiais e ocorre aumento na proliferação de raízes no local de maior disponibilidade de água.

Tabela 3. Densidade de raiz $\left(\mathrm{g} \mathrm{dm}^{-3}\right)$ de berinjela submetido a irrigação por gotejamento e sequeiro.

\begin{tabular}{|c|c|c|c|}
\hline \multirow{2}{*}{${ }^{1}$ Distância do caule } & \multirow{2}{*}{${ }^{2}$ Profundidade do solo } & Irrigado & Sequeiro \\
\hline & & \multicolumn{2}{|c|}{$\mathrm{g} \mathrm{dm}^{-3}$} \\
\hline \multirow{5}{*}{$\mathrm{D}_{1}$} & $\mathrm{P}_{1}$ & $* 6,21 \mathrm{a}$ & $1,81 b$ \\
\hline & $\mathrm{P}_{2}$ & $3,09 \mathrm{a}$ & $1,16 b$ \\
\hline & & & \\
\hline & $\mathrm{P}_{3}$ & $1,07 \mathrm{a}$ & $0,41 \mathrm{a}$ \\
\hline & $\mathrm{P}_{4}$ & $1,32 \mathrm{a}$ & $0,42 \mathrm{a}$ \\
\hline \multirow{4}{*}{$\mathrm{D}_{2}$} & $\mathrm{P}_{1}$ & $3,23 a$ & $1,09 b$ \\
\hline & $\mathrm{P}_{2}$ & $1,16 \mathrm{a}$ & $0,72 \mathrm{a}$ \\
\hline & $\mathrm{P}_{3}$ & $0,31 \mathrm{a}$ & $0,26 \mathrm{a}$ \\
\hline & $\mathrm{P}_{4}$ & $0,27 \mathrm{a}$ & $0,18 \mathrm{a}$ \\
\hline \multirow{4}{*}{$\mathrm{D}_{3}$} & $\mathrm{P}_{1}$ & $2,11 \mathrm{a}$ & $0,21 b$ \\
\hline & $\mathrm{P}_{2}$ & $1,07 \mathrm{a}$ & $0,13 b$ \\
\hline & $\mathrm{P}_{3}$ & $0,21 \mathrm{a}$ & $0,14 \mathrm{a}$ \\
\hline & $\mathrm{P}_{4}$ & $0,23 a$ & $0,08 \mathrm{a}$ \\
\hline
\end{tabular}

*Médias seguidas de letras iguais nas linhas não diferem entre si pelo teste $\mathrm{F}(\mathrm{P}>0,05)$

${ }^{1} \mathrm{D}_{1}=$ distância de zero metro do caule, $\mathrm{D}_{2}=$ distância 0,2 metro do caule, $\mathrm{D}_{3}=$ distancia de $0,4 \mathrm{~m}$ do caule. ${ }^{2} \mathrm{P} 1$ $=$ profundidade do solo de $0,00-0,10 \mathrm{~m}, \mathrm{P}_{2}=$ profundidade do solo de $0,10-0,20 \mathrm{~m}, \mathrm{P}_{3}=$ profundidade do solo de 0,20-0,30 $\mathrm{m}, \mathrm{P}_{4}=$ profundidade do solo de 0,30-0,40 $\mathrm{m}$.

A adubação química resultou no crescimento maior das raízes, principalmente na região mais próxima ao caule e na profundidade do solo $\mathrm{P}_{1}$, não havendo diferença significativa para outras distância do caule e profundidade do solo (Tabela 4). 


\section{DESENVOLVIMENTO RADICULAR DA BERINJELA IRRIGADO E DE SEQUEIRO EM DIFERENTES FORMAS DE CULTIVO}

Tabela 4. Densidade de raiz de $\left(\mathrm{g} \mathrm{dm}^{-3}\right)$ de berinjela em relação a adubação orgânica e química.

\begin{tabular}{|c|c|c|c|}
\hline \multirow{2}{*}{ Distância do caule } & \multirow{2}{*}{ Profundidade do solo } & Adubação orgânica & Adubação química \\
\hline & & \multicolumn{2}{|c|}{$\mathrm{g} \mathrm{dm}^{-3}$} \\
\hline \multirow{4}{*}{$\mathrm{D}_{1}$} & $\mathrm{P}_{1}$ & $* 3,00 \mathrm{~b}$ & $5,01 \mathrm{a}$ \\
\hline & $\mathrm{P}_{2}$ & $1,43 \mathrm{a}$ & $2,82 \mathrm{a}$ \\
\hline & $\mathrm{P}_{3}$ & $0,74 \mathrm{a}$ & $0,75 \mathrm{a}$ \\
\hline & $\mathrm{P}_{4}$ & $0,86 \mathrm{a}$ & $0,88 \mathrm{a}$ \\
\hline \multirow{4}{*}{$\mathrm{D}_{2}$} & $\mathrm{P}_{1}$ & $2,30 \mathrm{a}$ & $2,03 \mathrm{a}$ \\
\hline & $\mathrm{P}_{2}$ & $0,83 \mathrm{a}$ & $1,05 \mathrm{a}$ \\
\hline & $\mathrm{P}_{3}$ & $0,23 \mathrm{a}$ & $0,33 \mathrm{a}$ \\
\hline & $\mathrm{P}_{4}$ & $0,20 \mathrm{a}$ & $0,25 \mathrm{a}$ \\
\hline \multirow{4}{*}{$\mathrm{D}_{3}$} & $\mathrm{P}_{1}$ & $1,17 \mathrm{a}$ & $1,15 \mathrm{a}$ \\
\hline & $\mathrm{P}_{2}$ & $0,63 \mathrm{a}$ & $0,57 \mathrm{a}$ \\
\hline & $\mathrm{P}_{3}$ & $0,17 \mathrm{a}$ & $0,18 \mathrm{a}$ \\
\hline & $\mathrm{P}_{4}$ & $0,10 \mathrm{a}$ & $0,21 \mathrm{a}$ \\
\hline
\end{tabular}

*Médias seguidas de letras iguais nas linhas não diferem entre si pelo teste $\mathrm{F}(\mathrm{P}>0,05)$

${ }^{1} \mathrm{D}_{1}=$ distância de zero metro do caule, $\mathrm{D}_{2}=$ distância 0,2 metro do caule, $\mathrm{D}_{3}=$ distancia de $0,4 \mathrm{~m}$ do caule. ${ }^{2} \mathrm{P} 1$ $=$ profundidade do solo de $0,00-0,10 \mathrm{~m}, \mathrm{P}_{2}=$ profundidade do solo de $0,10-0,20 \mathrm{~m}, \mathrm{P}_{3}=$ profundidade do solo de $0,20-0,30 \mathrm{~m}, \mathrm{P}_{4}=$ profundidade do solo de $0,30-0,40 \mathrm{~m}$.

A concentração de raízes se manteve em uma distancia próxima com o caule, independente do sistema de adubação utilizada, concordando com Tecchio et al. (2005) que estudaram a distribuição do sistema radicular do maracujazeiro-doce com adubação orgânica e química, sendo que este apresentou concentração das raízes à uma distância de 0-20 cm do tronco.

Para a região de amostragem que teve diferença significativa, analisaram-se as médias de densidade de raiz de berinjela para a adubação orgânica, que não diferiu quanto ao irrigado e sequeiro, porém, quando comparada com a adubação química, suas médias foram superiores na presença de irrigação. Quando as adubações foram relacionadas entre si, estas não diferiram no sequeiro, porém, a adubação química mostrou-se superior na presença de irrigação (Tabela 5).

Tabela 5. Interação dos tratamentos de irrigação e adubação para a densidade de raiz $\left(\mathrm{g} \mathrm{dm}^{-3}\right)$ de berinjela na distância zero e profundidade de 0,00-0,10 m.

\begin{tabular}{ccc}
\hline Tratamentos & Irrigado & Sequeiro \\
\hline Adubação Orgânica & $* 4,23 \mathrm{bA}$ & $1,83 \mathrm{aA}$ \\
Adubação química & $8,19 \mathrm{aA}$ & $1,78 \mathrm{aB}$
\end{tabular}

*Médias seguidas de letras iguais minúscula na coluna e maiúscula na linha não diferem entre si pelo teste $\mathrm{F}$ $(\mathrm{P}>0,05)$.

Isto pode ser explicado pela alta resposta de crescimento das raízes com a absorção dos nutrientes prontamente disponíveis na adubação química, o que não ocorreu no sequeiro devido à falta de água que atua como campo de fluxo de 


\section{DESENVOLVIMENTO RADICULAR DA BERINJELA IRRIGADO E DE SEQUEIRO EM DIFERENTES FORMAS DE CULTIVO}

massa.

Encontra-se na Tabela 6, a porcentagem de raízes, no qual, verificouse que $82,08 \%$ da distribuição do sistema radicular da berinjela irrigado e utilizando adubação orgânica situa-se na profundidade $\mathrm{P}_{2}$ e, irrigado aplicando adubação química, encontrou-se 83,95\% para está mesma profundidade. Hunt, Morgan e Read (1998) relataram que a distribuição do sistema radicular no solo não é uniforme, sendo o movimento de água maior nas regiões do perfil onde as raízes estão mais concentradas.

Tabela 6. Porcentagem da distribuição das raízes de berinjela irrigado por gotejamento e sequeiro sob adubação orgânica e química, em distância do caule e profundidade do solo.

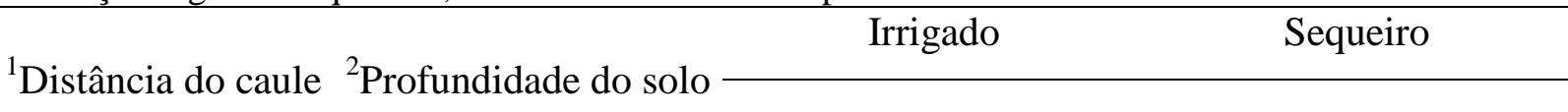

\begin{tabular}{|c|c|c|c|c|c|}
\hline \multicolumn{2}{|c|}{$\mathrm{m}$} & $* \mathrm{AO}$ & AQ & $\mathrm{AO}$ & AQ \\
\hline & & \multicolumn{4}{|c|}{$\%$} \\
\hline \multirow{4}{*}{$\mathrm{D}_{1}$} & $\mathrm{P}_{1}$ & 24,62 & 34,96 & 28,90 & 26,06 \\
\hline & $\mathrm{P}_{2}$ & 11,67 & 17,79 & 13,98 & 20,83 \\
\hline & $\mathrm{P}_{3}$ & 6,42 & 4,47 & 6,00 & 6,52 \\
\hline & $\mathrm{P}_{4}$ & 6,83 & 6,31 & 8,96 & 4,01 \\
\hline \multirow{4}{*}{$\mathrm{D}_{2}$} & $\mathrm{P}_{1}$ & 19,39 & 13,42 & 20,55 & 13,07 \\
\hline & $\mathrm{P}_{2}$ & 7,20 & 4,66 & 7,01 & 14,24 \\
\hline & $\mathrm{P}_{3}$ & 1,78 & 1,31 & 2,43 & 5,13 \\
\hline & $\mathrm{P}_{4}$ & 1,01 & 1,59 & 3,73 & 1,87 \\
\hline \multirow{4}{*}{$\mathrm{D}_{3}$} & $\mathrm{P}_{1}$ & 12,54 & 8,84 & 3,08 & 3,19 \\
\hline & $\mathrm{P}_{2}$ & 6,66 & 4,28 & 2,03 & 1,83 \\
\hline & $\mathrm{P}_{3}$ & 1,24 & 0,87 & 1,95 & 2,19 \\
\hline & $\mathrm{P}_{4}$ & 0,64 & 1,49 & 1,38 & 1,07 \\
\hline Total & - & 100 & 100 & 100 & 100 \\
\hline
\end{tabular}

*AO = Adubação orgânica; $\mathrm{AQ}=$ Adubação química. ${ }^{1} \mathrm{D}_{1}=$ distância de zero metro do caule, $\mathrm{D}_{2}=$ distância 0,2 metro do caule, $\mathrm{D}_{3}=$ distancia de $0,4 \mathrm{~m}$ do caule. ${ }^{2} \mathrm{P} 1=$ profundidade do solo de $0,00-0,10 \mathrm{~m}, \mathrm{P}_{2}=$ profundidade do solo de $0,10-0,20 \mathrm{~m}, \mathrm{P}_{3}=$ profundidade do solo de $0,20-0,30 \mathrm{~m}, \mathrm{P}_{4}=$ profundidade do solo de $0,30-0,40 \mathrm{~m}$.

Com o tratamento de sequeiro observou-se que, apesar da menor quantidade de massa seca de raiz, tanto o tratamento com adubação orgânica e química alcançaram os valores de 74,65\% e $79,22 \%$ respectivamente até a profundidade $\mathrm{P}_{2}$. Pinto et al. (2008), relataram que houve redução significativa da matéria seca de raiz na cultura do amendoim, gergelim e mamona quando submetidas a deficiência hídrica.Verificase também que na distância do caule zero a densidade de raiz até a profundidade de $0,40 \mathrm{~m}$ é de $49,54 \%$ e $63,53 \%$ para a berinjela irrigada por gotejamento com adubação orgânica e química respectivamente.

Os modelos de superfície de 


\section{DESENVOLVIMENTO RADICULAR DA BERINJELA IRRIGADO E DE SEQUEIRO EM DIFERENTES FORMAS DE CULTIVO}

resposta testados para estimar a densidade de raiz de berinjela irrigado utilizando adubação orgânica, o que melhor se ajustou aos dados obtidos na pesquisa, foi à combinação de modelo quadrático com os parâmetros de $\mathrm{D}$ e $\mathrm{P}$, apresentando coeficiente de determinação $\left(\mathrm{R}^{2}\right)$ igual a 0,70 (Tabela 7). Os resultados do teste $t$ demonstraram efeitos significativos para o parâmetro $\mathrm{D}(\operatorname{Pr}>|\mathrm{t}|=0,0148)$, altamente significativo para a parâmetro $\mathrm{P}$ $(\operatorname{Pr}>|\mathrm{t}|=0,0001)$, significativo para a variável $\left.\mathrm{P}^{2} \mathrm{Pr}>|\mathrm{t}|=0,0067\right)$. O modelo de superfície de resposta para estimar a densidade de raiz de berinjela irrigada utilizando adubação química obteve $\mathrm{R}^{2}$ de 0,80 , sendo que as variáveis da equação $\mathrm{D}$, $\mathrm{P}$ e DP mostraram efeito altamente significativo $(\operatorname{Pr}>|t|=0,0001)$ e as variáveis $\mathrm{D}^{2}$ e $\mathrm{P}^{2}$ foram significativo com $\operatorname{Pr}>|\mathrm{t}|=0,0032$ e 0,0003 respectivamente.

Tabela 7. Equações de superfície de resposta de densidade de raiz $\left(\mathrm{g} \mathrm{dm}^{-3}\right)$ de berinjela (Solanum melongena L.) irrigado por gotejamento e sequeiro sob adubação orgânica e químico.

\begin{tabular}{|c|c|c|c|c|}
\hline \multicolumn{2}{|c|}{ *Tratamentos } & Equações de superfície de resposta & $\mathrm{R}^{2}$ & $\operatorname{Pr}>\mathrm{F}$ \\
\hline \multirow{2}{*}{ Irrigado } & $\mathrm{AO}$ & $\hat{\mathrm{Y}}=5,44-7,10 \mathrm{D}-27,87 \mathrm{P}+43,00 \mathrm{P}^{2}$ & 0,70 & 0,0001 \\
\hline & AQ & $\hat{\mathrm{Y}}=9,79-26,54 \mathrm{D}-47,39 \mathrm{P}+27,15 \mathrm{D}^{2}+64,31 \mathrm{P}^{2}+43,24 \mathrm{DP}$ & 0,80 & 0,0001 \\
\hline \multirow{2}{*}{ Sequeiro } & $\mathrm{AO}$ & $\hat{\mathrm{Y}}=2,27-3,75 \mathrm{D}-11,39 \mathrm{P}+17,06 \mathrm{P}^{2}+9,66 \mathrm{DP}$ & 0,62 & 0,0001 \\
\hline & $\mathrm{AQ}$ & $\hat{\mathrm{Y}}=2,11-4,52 \mathrm{D}-5,29 \mathrm{P}+13,08 \mathrm{DP}$ & 0,73 & 0,0001 \\
\hline
\end{tabular}

*AO = Adubação orgânica; AQ = Adubação química; $\mathrm{D}$ = Distância do caule; $\mathrm{P}$ = Profundidade do solo.

O modelo para estimar a densidade de raiz sem irrigação com adubação orgânica obteve $\mathrm{R}^{2}$ igual a 0,62 , no qual os parâmetros da equação demonstraram efeito significativo para o parâmetro D ( $\mathrm{Pr}$ $>|\mathrm{t}|=0,0250)$, altamente significativo para a parâmetro $\mathrm{P}(\mathrm{Pr}>|\mathrm{t}|=0,0001)$, significativo para a variável $\mathrm{P}^{2}(\operatorname{Pr}>|\mathrm{t}|=$ $0,0012)$ e a variável DP significativo com $\operatorname{Pr}>|\mathrm{t}|=0,0052$.

Para a densidade de raiz sem irrigação com adubação química o modelo foi representado apenas com parâmetros lineares, no qual, a variável $\mathrm{D}$ foi significativo pelo teste $\mathrm{t}$ com $\operatorname{Pr}>|\mathrm{t}|=$ 0,0009 , a variável $P$ foi significativo com $\operatorname{Pr}>|\mathrm{t}|=0,0190$ e produto cruzado DP significativo com $\operatorname{Pr}>|t|=0,0001$.

Observa-se na Figura 1A, a representação gráfica de superfície de resposta da densidade de raiz em relação à distância do caule e profundidade do solo com berinjela irrigada por gotejamento com adubação orgânica, evidenciando que a densidade de raízes decresce com o aumento da distância do caule e profundidade do solo.

No entanto verificou-se que essa evidencia tem efeito significativo mais alto no fator profundidade do solo sobre a densidade de raiz de berinjela. Tal análise pode ser observada face aos gradientes de declive das linhas, que compõem a superfície de resposta, que apresentou-se mais acentuados para profundidade do solo do que o fator distância do caule, que em distancia zero do caule e profundidade $\mathrm{P}_{1}$, tem se o valor de densidade de raiz de 4,16 $\mathrm{g} \mathrm{dm}^{-3}$ (Figura 1A). 


\section{DESENVOLVIMENTO RADICULAR DA BERINJELA IRRIGADO E DE SEQUEIRO EM DIFERENTES FORMAS DE CULTIVO}
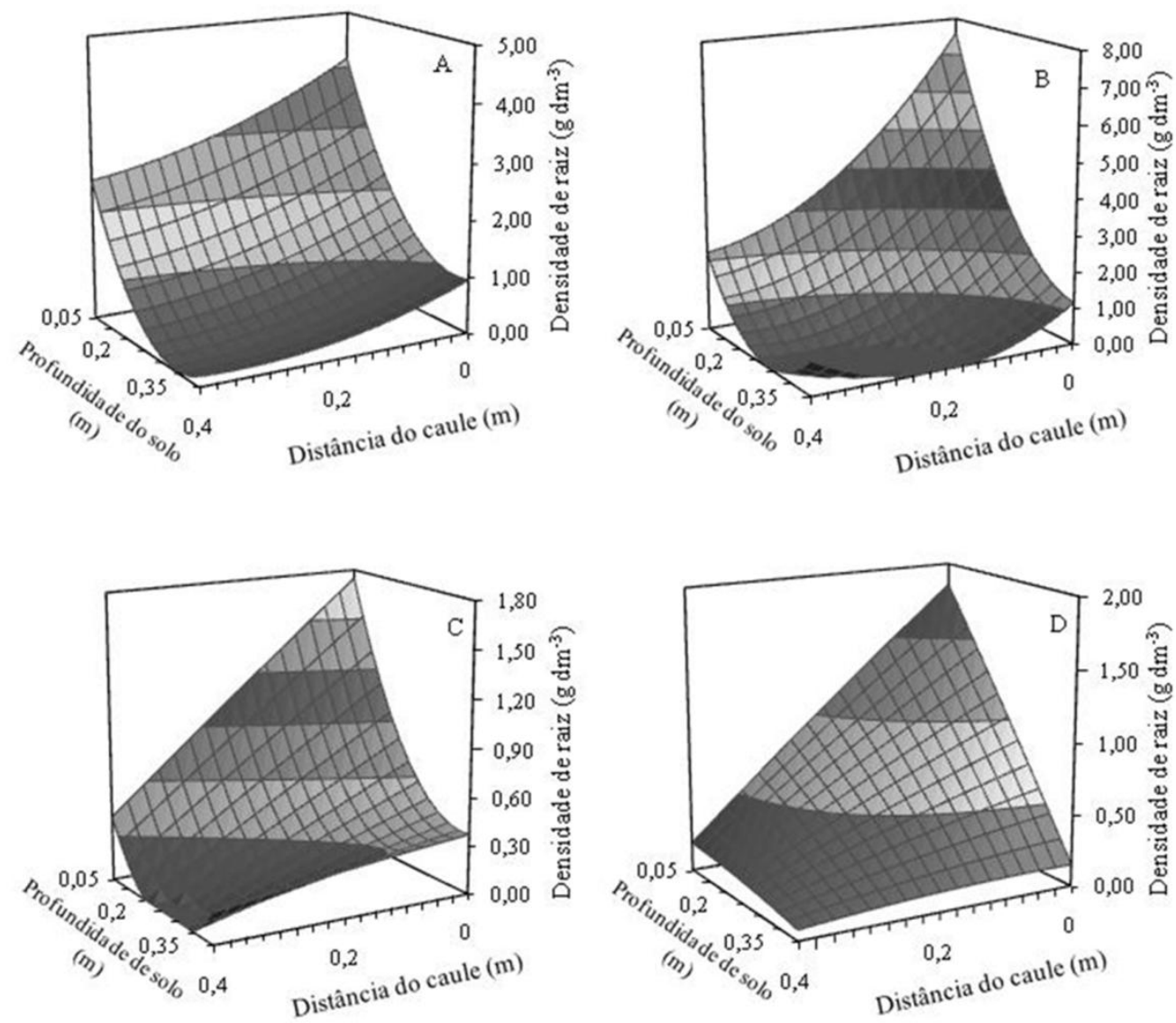

Figura 1. Superfície de resposta da densidade de raiz da berinjela irrigado por gotejamento aplicando adubação orgânica (A) e adubação química (B) e, sequeiro aplicando adubação orgânica (C) e adubação química (D) em relação à distância do caule e profundidade do solo.

$\mathrm{Na}$ Figura 1B, observa-se a regressão de superfície de resposta da densidade de raiz da berinjela irrigado por gotejamento usando adubação química em relação distância do caule e profundidade do solo que, estima na distância zero do caule e profundidade do solo $\mathrm{P}_{1}$, o valor de $7,58 \mathrm{~g} \mathrm{dm}^{-3}$. Esse valor representa uma variação de $3,43 \mathrm{~g} \mathrm{dm}^{-3}$ de densidade raiz para essa mesma distância do caule e profundidade do solo quando comparada com a raiz da berinjela irrigada com adubação orgânica. Nestas condições, o manejo da irrigação deve prevalecer adotando-se turnos de rega mais freqüentes para que não haja a percolação da água no perfil do solo e, portanto acúmulo de água abaixo da profundidade efetiva do sistema radicular.

Encontra-se na Figura 1C e 1D, a representação gráfica da densidade de raiz de berinjela de sequeiro com adubação orgânica e química respectivamente. Ainda que os sistemas radiculares das plantas de berinjela sejam muito superficiais, verificase que sem irrigação houve uma melhor distribuição das raízes, em contraste com a menor densidade de raiz.

Braga et al. (1999) relataram, que a distribuição do sistema radicular em profundidade, devido a insuficiência hídrica, é considerada como parâmetro indicador de tolerância a seca e pode conferir adaptação em algumas espécies.

Nas condições de berinjela irrigada por gotejamento, entende-se que o manejo 


\section{DESENVOLVIMENTO RADICULAR DA BERINJELA IRRIGADO E DE SEQUEIRO EM DIFERENTES FORMAS DE CULTIVO}

da irrigação deve prevalecer para que tenha-se maior distribuição e densidade de raiz adotando-se turnos de rega mais freqüentes para que não haja a percolação

\section{CONCLUSÕES}

A ausência de irrigação resulta em uma diminuição significativa da densidade de raiz da berinjela, comparada com berinjela irrigada. Apenas na profundidade de $0,1 \mathrm{~m}$ há influencia da associação da

\section{REFERÊNCIAS}

BERNARDO, S.; SOARES, A.A.; MANTOVANI, E.C. Manual de irrigação. $8^{\mathrm{a}}$ ed.Viçosa: UFV, 2008. 625p.

BRAGA, M.B.; RAMOS, M.M.; GOMIDE, R.L. Influencia de quatro frequiências de irrigação na distribuição radicular, em três estádios de desenvolviemnto da cultura do feijoeiro (Phaseolus vulgaris L. cv. Carioca). Irriga, Botucatu, v. 04, n. 03, p.175-182, 1999.

COELHO, E. F.; SANTOS, M.R.; COELHO FILHO, M. A. Distribuição de raízes de mamoeiro sob diferentes sistemas de irrigação localizada em Latossolo de tabuleiros costeiros. Revista Brasileira de Fruticultura, Jaboticabal, v. 27, n.1, p. 175-178, 2005.

EMPRESA BRASILEIRA DE PESQUISA AGROPECUÁRIA - EMBRAPA. Centro Nacional de Pesquisa em Solos. Sistema brasileiro de classificação de solos. 2.ed. Rio de Janeiro, 2006. 306p.

EMPRESA BRASILEIRA DE PESQUISA AGROPECUÁRIA - EMBRAPA. Centro Nacional de Pesquisa em Solos. Manual de métodos de análise de solo. 2.ed. Rio de Janeiro, 1997. 212p. da água no perfil do solo e portanto, acúmulo de água abaixo da profundidade efetiva do sistema radicular.

irrigação e da adubação química e orgânica na densidade de raiz. Recomenda-se que a profundidade efetiva do sistema radicular da berinjela irrigada por gotejamento, com aplicação de adubação orgânica e química seja no máximo de 0,20 metros.

FILGUEIRA, F.A.R. Novo manual de olericultura: agrotecnologia moderna, produção e comercialização de hortaliças. Viçosa: UFV, 2000. 402p.

FILGUEIRA, F.A.R. Solanáceas: agroecologia moderna na produção de tomate, batata, pimentão, berinjela $e$ jiló. Lavras: UFLA, 2003. 333p.

FRACARO, A. A.; PEREIRA, F. M. Distribuição do sistema radicular da goiabeira 'Rica' produzida a partir de estaquia herbácea. Revista Brasileira de Fruticultura, Jaboticabal, v. 26, n. 1, p. 183-195, 2004.

HUNT, H. W.; MORGAN, J. A.; READ, J. J. Simulating growth and root-shoot partitioning in prairie grasses under elevated atmospheric $\mathrm{CO}_{2}$ and water stress. Annals of Botany, London, v. 81, n. 4, p. 489-501, 1998.

MIYASAKA, S.; NAKAMURA, Y.; OKAMOTO, H. Agricultura natural. 2. ed. Cuiabá: SEBRAE/MT, 1997. 73p.

PINTO, C.M.; TAVORA, F.J.F.A.; BEZERRA, M.A.; CORRÊA, M.C.M. Crescimento, distribuição do sistema radicular em amendoim, gergelim e mamona a ciclos de deficiência hídrica.

Revista Ciência Agronômica, Fortaleza, v. 49, n. 3, p. 429-436, 2008. 
RIBEIRO, C.S.C.; BRUNE, S.; REIFCHNEIDER, F.J.B. Cultivo da berinjela. Brasília: Embrapa Hortaliças, 1998. 23 p. (Embrapa Hortaliças. Instrução Técnica 15).

SAS INSTITUTE. SAS/STAT. Users Guide: Version 8. Cary: SAS Institute Inc., 1999. 3809p.
TAIZ, L.; ZEIGER, E. Fisiologia vegetal. 3. ed. Porto Alegre: Artmed, 2004. 719 p.

TECCHIO, M.A.; DAMATTO JUNIOR, E.R.; LEONEL, S.; PEDROSO, C. J. Distribuição do sistema radicular do maracujazeiro-doce cultivado com adubação química e orgânica. Revista Brasileira de Fruticultura, Jaboticabal, v. 27, n. 2, p. 324-326, 2005. 